\title{
Epidemic Model of Leptospirosis Containing Fractional Order
}

\author{
Muhammad Altaf Khan, ${ }^{1}$ S. F. Saddiq, ${ }^{2}$ Saeed Islam, ${ }^{1}$ \\ Ilyas Khan, ${ }^{3}$ and Dennis Ling Chuan Ching ${ }^{4}$ \\ ${ }^{1}$ Department of Mathematics, Abdul Wali Khan University, Mardan, Khyber Pakhtunkhwa, Pakistan \\ ${ }^{2}$ Department of Mathematics, Islamia College University, Peshawar, Khyber Pakhtunkhwa, Pakistan \\ ${ }^{3}$ College of Engineering, Majmaah University, Majmaah, Saudi Arabia \\ ${ }^{4}$ Department of Fundamental Applied Sciences, Universiti Teknologi PETRONAS, 31750 Perak, Malaysia
}

Correspondence should be addressed to Dennis Ling Chuan Ching; dennis.ling@petronas.com.my

Received 23 July 2014; Revised 21 September 2014; Accepted 24 September 2014; Published 11 December 2014

Academic Editor: Yasir Khan

Copyright (C) 2014 Muhammad Altaf Khan et al. This is an open access article distributed under the Creative Commons Attribution License, which permits unrestricted use, distribution, and reproduction in any medium, provided the original work is properly cited.

We study an epidemic model of leptospirosis in fractional order numerically. The multistep generalized differential transform method is applied to find the accurate approximate solution of the epidemic model of leptospirosis disease in fractional order. A unique positive solution for the epidemic model in fractional order is presented. For the integer case derivative, the approximate solution of MGDTM is compared with the Runge-Kutta order four scheme. The numerical results are presented for the justification purpose.

\section{Introduction}

Leptospirosis disease is an important infectious disease. This kind of infection occurs in urban areas of industrialized and developed countries and also in the rural areas. The people of the city who walk in dirty water are mostly infected. Workers planting rice, sewer cleaners, cleaning canals workers, and agriculture labor get the disease easily. The disease flourishes due to delay in diagnosis and unavailability of clinical infrastructure. The cause of the disease is bacteria. It is potentially fatal infection of brain, kidney, liver, heart, and lung. The people who can get infection are those who have contact with infected animals, soil, or water in which the bacteria is present. The outdoor people, who work with animals, face the risk of leptospirosis infection, similarly workers in farms, sewer, mine, slaughter houses, dairy farmers, and animal caretakers and those who work with fishes and military personnel. Those people who work outdoors like swimming, rafting, and kayaking also face the risk of infection [1].

The mathematical modeling dealing with the complex biological structures is a great challenge for the researchers. For simple biological models, the study of integer order differential equations is enough for the description of their dynamics while for those systems contains complexities are characterized due to its variability structured properties, like nonlinearity of the systems, multiscale behavior, and the mathematical relation between the parameters [2]. To study such nonlinear biological models, with complex mathematical structures, the fractional derivatives provide the tool to handle the dynamical behavior of such complex systems. The fundamental property related to these models is the nonexistence of the differential operator of integer order. These characteristics show information about the present but also show its historical states.

The fractional derivative and fractional integrals were first used by Magin [3] in his model which is related to the relation between stress-strain and biomaterials. A new fractional analytical approach via a modified Riemann Liouville derivative has been used by [4]. For solution of the 2D region with obstacles, the author [5] used the fractional differential equations. For the giving up smoking model, the fractional differential equation was used by [6]. The author used the differential transforms method and Pade approximants for a fractional population growth model [7]. For more information about fractional calculus, see $[2,8-10]$. The author had made many efforts for modeling of leptospirosis epidemic disease since 2011; see [11-16]. The author proposed 
a mathematical model that describes the epidemic leptospirosis disease [11]. The model proposed by [11] is given by

$$
\begin{gathered}
\frac{d S^{h}}{d t}=b_{1}-\mu_{h} S^{h}-\beta_{2} S^{h} I^{v}-\beta_{1} S^{h} I^{h}+\lambda_{h} R^{h}, \\
\frac{d I^{h}}{d t}=\beta_{2} S^{h} I^{v}+\beta_{1} S^{h} I^{h}-\left(\mu_{h}+\delta_{h}+\gamma_{h}\right) I^{h}, \\
\frac{d R^{h}}{d t}=\gamma_{h} I^{h}-\mu_{h} R^{h}-\lambda^{h} R^{h}, \\
\frac{d S^{v}}{d t}=b_{2}-\gamma_{v} S^{v}-\beta_{3} S^{v} I^{h}, \\
\frac{d I^{v}}{d t}=\beta_{3} S^{v} I^{h}-\left(\gamma_{v}+\delta_{v}\right) I^{v},
\end{gathered}
$$

and the related initial conditions:

$$
\begin{array}{ll}
S^{h}(0)=e_{1}, & I^{h}(0)=e_{2}, \quad R^{h}(0)=e_{3}, \\
S^{v}(0)=e_{4}, & I^{v}(0)=e_{5} .
\end{array}
$$

Here $S^{h}(t), I^{h}(t), R^{h}(t), S^{v}(t)$, and $I^{v}(t)$, respectively, represent the population of susceptible human, infected human, recovered human, susceptible vector, and infected vector at time $t$. The rate at which the population of human increases is shown by $b_{1}$. The natural mortality rate for the human population is $\mu_{h} ; \beta_{1}, \beta_{2}$, and $\beta_{3}$ represent the transmission coefficients. The parameter $\lambda_{h}$ shows the individuals who become susceptible again. The death from the disease that occurs to humans is shown by $\delta_{h}$. The rate of recovery from infection for the human is denoted by $\gamma_{h}$. The growth rate of the vector population is represented by $b_{2} ; \gamma_{v}$ is the natural death rate for vector and disease related death rate for the vector is $\delta_{v}$.

The model presented by [11] is given by system (1) which represents a system of nonlinear ODE; their fractional order differential equation is given as follows:

$$
\begin{gathered}
D_{t}^{\alpha} S^{h}(t)=b_{1}-\mu_{h} S^{h}-\beta_{2} S^{h} I^{v}-\beta_{1} S^{h} I^{h}+\lambda_{h} R^{h}, \\
D_{t}^{\alpha} I^{h}(t)=\beta_{2} S^{h} I^{v}+\beta_{1} S^{h} I^{h}-\left(\mu_{h}+\delta_{h}+\gamma_{h}\right) I^{h}, \\
D_{t}^{\alpha} R^{h}(t)=\gamma_{h} I^{h}-\mu_{h} R^{h}-\lambda^{h} R^{h}, \\
D_{t}^{\alpha} S^{v}(t)=b_{2}-\gamma_{v} S^{v}-\beta_{3} S^{v} I^{h}, \\
D_{t}^{\alpha} I^{v}(t)=\beta_{3} S^{v} I^{h}-\left(\gamma_{v}+\delta_{v}\right) I^{v},
\end{gathered}
$$

where $D_{t}^{\alpha}$ represents the fractional derivative in Caputo derivative sense and the order of the fractional derivative is shown by the parameter $\alpha$ and $0<\alpha<1$, with the related initial conditions (2). The parameter which describes the general response expression in representing the order of fractional derivative gives different results for different values. Obviously, the integer-order system can be viewed as a special case of the fractional-order system by putting the time-fractional order of the derivative equal to one. To put it simple, for the higher in order, the behavior of the fractional order system is the same in the case of integer order. According to research, this is the first work, which is available on the epidemic model of leptospirosis in fractional order. Due to this reason, we express the approximate solutions to the problems (3) by the technique of (MSGDTM). The solution to such nonlinear fractional equation can also be available by using an efficient approach [17].

The MSGDTM is a modified form of the GDTM $[6,8,18-$ $20]$, in which it is treated as an algorithm in a sequence of small steps, to obtain the accurate approximate solution to the desire models. By using GDTM, the obtained solution is valid for short interval of time while the solution obtained from multistep generalized differential transform method (MSGDTM) is valid for a long time. To obtain the solution, using MSGDTM is more accurate and valid for long interval of time and agrees well with the classical Runge-Kutta numerical solution method, with the unity order derivative $[6,21]$.

The paper has been organized/structured as follows. Section 1 is introduction; Section 2 contains basic definitions of fractional calculus and their notations; Section 3 gives nonnegative solution of the model; Section 4 contains application of MSGDTM; Section 5 gives numerical results; and Section 6 is the conclusion.

\section{Basic Definitions and Notations}

This section contains some fundamental definitions and notations of fractional calculus that will help us in the proceeding sections $[3,22-24]$.

Definition 1. A function $g(x)$ with the positive values of $x$ is known in the space $C_{\alpha}(\alpha \in R)$ if it is written in the form $g(x)=x^{q} g_{1}(x)$ and for some $q>\alpha$, where $g_{1}(x)$ is continuous in $[0, \infty)$, and it is known to be in the space $C_{\alpha}^{n}$ if $g^{(n)} \in C_{\alpha}, n \in \mathbf{N}$.

Definition 2. The Riemann Liouville integral operator of order $\alpha>0$ with $a \geq 0$ is defined as

$$
\begin{gathered}
\left(J_{a}^{\alpha} g\right)(x)=\frac{1}{\Gamma(\alpha)} \int_{a}^{x}(x-t)^{\alpha-1} g(t) d t, \quad x>\alpha, \\
\left(J_{a}^{0} g\right)(x)=g(x) .
\end{gathered}
$$

For the properties of the operator [22], we need only the following. For $g \in C_{\alpha}, \alpha>0, \beta>0, c \in R$, and $\gamma>-1$, one has

$$
\begin{gathered}
\left(J_{a}^{\alpha} J_{a}^{\beta} g\right)(x)=\left(J_{a}^{\beta} J_{a}^{\alpha} g\right)(x)=\left(J_{a}^{\alpha+\beta} g\right)(x), \\
J_{\alpha}^{\alpha} x^{\gamma}=\frac{x^{\gamma+\alpha}}{\Gamma(\alpha)} B_{(x-a) / x}(\alpha, \gamma+1),
\end{gathered}
$$

where $B_{\tau}(\alpha, \gamma+1)$ represents the incomplete beta function defined as

$$
\begin{gathered}
B_{\tau}(\alpha, \gamma+1)=\int_{0}^{\tau} t^{\alpha_{1}}(1-t)^{\gamma} d t \\
J_{a}^{\alpha} e^{c x}=e^{a c}(x-a)^{\alpha} \sum_{k=0}^{\infty} \frac{[c(x-a)]^{k}}{\Gamma(\alpha+k+1)} .
\end{gathered}
$$


The Riemann Liouville derivative has some disadvantages when applying to real world model with fractional differential equations. Therefore, here we use a modified fractional differential operator $D_{a}^{\alpha}$ which is used in his work by Caputo on the theory of viscoelasticity.

Definition 3. The Caputo fractional derivative of $g(x)$ of order $\alpha>0$ with $a \geq 0$ is defined as

$$
\begin{aligned}
\left(D_{a}^{\alpha} g\right)(x) & =\left(J_{a}^{m-\alpha} g^{(m)}\right)(x) \\
& =\frac{1}{\Gamma(m-\alpha)} \int_{a}^{x} \frac{g^{(m)}(t)}{(x-t)^{(\alpha+1-m)}} d t
\end{aligned}
$$

for $m-1<\alpha \leq m, m \in N, x \geq a, f(x) \in C_{-1}^{m}$. Many authors investigated the Caputo fractional order derivative; for $m-1<\alpha \leq m, g(x) \in C_{\alpha}^{m}$, and $\alpha \geq-1$, one gets

$$
\left(J_{a}^{\alpha} D_{a}^{\alpha} g\right)(x)=J^{(m)} D^{(m)} g(x)=g(x)-\sum_{k=0}^{m-1} g^{(k)}(a) \frac{(x-a)^{k}}{k !} .
$$

\section{Nonnegativity of the Model}

Suppose $R_{+}^{5}=\left\{X^{\natural} \in R^{5}: X^{\natural} \geq 0\right\}$ and $X^{\natural}(t)=\left(S_{h}(t)\right.$, $\left.I_{h}(t), R_{h}(t), S_{v}(t), I_{v}(t)\right)^{T}$. To prove the theorem, first, we state the following lemma [25].

Lemma 4. Suppose $g(x) \in C[a, b]$ and $D^{\alpha} g(x) \in C[a, b]$ for $\alpha \in(0,1]$. Then one has

$$
g(x)=g(a)+\frac{1}{\Gamma(\alpha)} D^{\alpha} g(\eta)(x-a)^{\eta},
$$

with $0 \leq \eta \leq x$, for all $x \in[a, b]$. This is also called generalized mean value theorem.

Remark 5. Suppose $g(x) \in C[a, b]$ and $D^{\alpha} g(x) \in C[a, b]$ for $0<\alpha \leq 1$. It is obvious from Lemma 4 that if $D^{\alpha} g(x) \geq$ $0 \forall x \in(0, b)$, then the $g$ is nonincreasing function.

Theorem 6. For the initial value problems (3), a unique solution exists and remains in $R_{+}^{5}$.

Proof. The existence and uniqueness of the solution of (3) in $(0, \infty)$ can be obtained from [26]. We just need to prove that the domain $R_{+}^{5}$ is positive invariant. Since

$$
\begin{gathered}
\left.D_{t}^{\alpha} S^{h}(t)\right|_{S^{h}=0}=b_{1}+\lambda_{h} R^{h}(t) \geq 0, \\
\left.D_{t}^{\alpha} I^{h}(t)\right|_{I^{h}=0}=\beta_{2} S^{h}(t) I^{v}(t) \geq 0, \\
\left.D_{t}^{\alpha} R^{h}(t)\right|_{R^{h}=0}=\gamma_{h} I^{h}(t) \geq 0, \\
\left.D_{t}^{\alpha} S^{v}(t)\right|_{S^{v}=0}=b_{2} \geq 0, \\
\left.D_{t}^{\alpha} I^{v}(t)\right|_{I^{v}=0}=\beta_{3} S^{v}(t) I^{h}(t) \geq 0,
\end{gathered}
$$

on each hyperplane bounding the nonnegative orthant, the vector field points into $R_{+}^{5}$.

\section{MSGDTM: Its Application}

The general differences transform method (GDTM) is used to provide approximate solutions for nonlinear problems with respect to the convergent series with easily computable components. It has shown that the solution obtained is estimated to be invalid for large $t$ for some systems [21, 27]. We therefore use multistep generalized differential transform method (MSGDTM) to approximate the solution of (3), which provides accurate solution for a period that is longer than the standard generalized difference transform method $[21,27]$. Several authors used the MSGDTM; see, for example, $[28,29]$. We take the differential transform of (3) with respect to time $t$ as

$$
\begin{aligned}
& S^{h}(k+1) \\
& =\frac{\Gamma(\alpha k+1)}{\Gamma(\alpha(k+1)+1)} \\
& \times\left(b_{1}-\sum_{k_{2}=0}^{k} \sum_{k_{1}=0}^{k_{2}} B_{2}\left(k_{1}\right) S^{h}\left(k_{2}-k_{1}\right) I^{v}\left(k-k_{2}\right)\right. \\
& -\mu_{h} S^{h}(k)+\lambda_{h} R^{h}(k) \\
& \left.-\sum_{k_{2}=0}^{k} \sum_{k_{1}=0}^{k_{2}} B_{1}\left(k_{1}\right) S^{h}\left(k_{2}-k_{1}\right) I^{h}\left(k-k_{2}\right)\right) \text {, } \\
& I^{h}(k+1) \\
& =\frac{\Gamma(\alpha k+1)}{\Gamma(\alpha(k+1)+1)} \\
& \times\left(\sum_{k_{2}=0}^{k} \sum_{k_{1}=0}^{k_{2}} B_{2}\left(k_{1}\right) S^{h}\left(k_{2}-k_{1}\right) I^{v}\left(k-k_{2}\right)\right. \\
& -\left(\mu_{h}+\delta_{h}+\gamma_{h}\right) I^{h}(k) \\
& \left.+\sum_{k_{2}=0}^{k} \sum_{k_{1}=0}^{k_{2}} B_{1}\left(k_{1}\right) S^{h}\left(k_{2}-k_{1}\right) I^{h}\left(k-k_{2}\right)\right) \text {, } \\
& R^{h}(k+1)=\frac{\Gamma(\alpha k+1)}{\Gamma(\alpha(k+1)+1)}\left(\gamma_{h} I^{h}(k)-\left(\mu_{h}+\lambda_{h}\right) R^{h}(k)\right), \\
& S_{v}(k+1)=\frac{\Gamma(\alpha k+1)}{\Gamma(\alpha(k+1)+1)} \\
& \times\left(b_{2}-\gamma_{v} S^{v}(k)\right. \\
& \left.-\sum_{k_{2}=0}^{k} \sum_{k_{1}=0}^{k_{2}} B_{3}\left(k_{1}\right) S^{v}\left(k_{2}-k_{1}\right) I^{h}\left(k-k_{2}\right)\right), \\
& I_{v}(k+1)=\frac{\Gamma(\alpha k+1)}{\Gamma(\alpha(k+1)+1)} \\
& \times\left(\sum_{k_{2}=0}^{k} \sum_{k_{1}=0}^{k_{2}} B_{3}\left(k_{1}\right) S^{v}\left(k_{2}-k_{1}\right) I^{h}\left(k-k_{2}\right)\right. \\
& \left.-\left(\gamma_{v}+\delta_{v}\right) I^{v}(k)\right)
\end{aligned}
$$


where $S^{h}(k), I^{h}(k), R^{h}(k), S^{v}(k), I^{v}(k), B_{1}(k), B_{2}(k)$, and $B_{3}(k)$ represent the differential transformations of $S^{h}(t), I^{h}(t)$, $R^{h}(t), S^{v}(t), I_{v}(t), \beta_{1}(t), \beta_{2}(t)$, and $\beta_{3}(t)$. The initial conditions in terms of differential transform form are represented as $S^{h}(0)=e_{1}, I^{h}(0)=e_{2}, R^{h}(0)=e_{3}, S^{v}(0)=e_{4}$, and $I^{v}(0)=e_{5}$. From differential inverse transforms point of view, the differential transform series solution for the systems (3) can be obtained as

$$
\begin{aligned}
& S^{h}(t)=\sum_{k=0}^{K} S^{h}(k) t^{\alpha k}, \\
& I^{h}(t)=\sum_{k=0}^{K} I^{h}(k) t^{\alpha k}, \\
& R^{h}(t)=\sum_{k=0}^{K} R^{h}(k) t^{\alpha k}, \\
& S^{v}(t)=\sum_{k=0}^{K} S^{v}(k) t^{\alpha k}, \\
& I^{v}(t)=\sum_{k=0}^{K} I^{v}(k) t^{\alpha k} .
\end{aligned}
$$

The series solution for the systems (3), according to MSGDTM is suggested as

$$
\begin{aligned}
& S^{h}(t)= \begin{cases}\sum_{k=0}^{K} S_{1}^{h}(k) t^{\alpha k}, & t \in\left[0, t_{1}\right] \\
\sum_{k=0}^{K} S_{2}^{h}(k)\left(t-t_{1}\right)^{\alpha k}, & t \in\left[t_{1}, t_{2}\right] \\
\vdots & \\
\sum_{k=0}^{K} S_{M}^{h}(k)\left(t_{M}-t_{M-1}\right)^{\alpha k}, & t \in\left[t_{M-1}, t_{M}\right],\end{cases} \\
& I^{h}(t)= \begin{cases}\sum_{k=0}^{K} I_{1}^{h}(k) t^{\alpha k}, & t \in\left[0, t_{1}\right] \\
\sum_{k=0}^{K} I_{2}^{h}(k)\left(t-t_{1}\right)^{\alpha k}, & t \in\left[t_{1}, t_{2}\right] \\
\vdots & \\
\sum_{k=0}^{K} I_{M}^{h}(k)\left(t_{M}-t_{M-1}\right)^{\alpha k}, & t \in\left[t_{M-1}, t_{M}\right],\end{cases} \\
& R^{h}(t)= \begin{cases}\sum_{k=0}^{K} R_{1}^{h}(k) t^{\alpha k}, & t \in\left[0, t_{1}\right] \\
\sum_{k=0}^{K} R_{2}^{h}(k)\left(t-t_{1}\right)^{\alpha k}, & t \in\left[t_{1}, t_{2}\right] \\
\vdots & \\
\sum_{k=0}^{K} R_{M}^{h}(k)\left(t_{M}-t_{M-1}\right)^{\alpha k}, & t \in\left[t_{M-1}, t_{M}\right],\end{cases}
\end{aligned}
$$$$
S^{v}(t)= \begin{cases}\sum_{k=0}^{K} S_{1}^{v}(k) t^{\alpha k}, & t \in\left[0, t_{1}\right] \\ \sum_{k=0}^{K} S_{2}^{v}(k)\left(t-t_{1}\right)^{\alpha k}, & t \in\left[t_{1}, t_{2}\right] \\ \vdots & \\ \sum_{k=0}^{K} S_{M}^{v}(k)\left(t_{M}-t_{M-1}\right)^{\alpha k}, & t \in\left[t_{M-1}, t_{M}\right],\end{cases}
$$$$
I^{v}(t)= \begin{cases}\sum_{k=0}^{K} I_{1}^{v}(k) t^{\alpha k}, & t \in\left[0, t_{1}\right] \\ \sum_{k=0}^{K} I_{2}^{v}(k)\left(t-t_{1}\right)^{\alpha k}, & t \in\left[t_{1}, t_{2}\right] \\ \vdots & \\ \sum_{k=0}^{K} I_{M}^{v}(k)\left(t_{M}-t_{M-1}\right)^{\alpha k}, & t \in\left[t_{M-1}, t_{M}\right],\end{cases}
$$

where $S_{i}^{h}(k), I_{i}^{h}(k), R_{i}^{h}(k), S_{i}^{v}(k)$, and $I_{i}^{v}(k)$ for $i=1,2$, $3, \ldots, M$ satisfy the following recurrence relations:

$$
S_{i}^{h}(k+1)
$$

$$
\begin{aligned}
& =\frac{\Gamma(\alpha k+1)}{\Gamma(\alpha(k+1)+1)} \\
& \times\left(b_{1}-\sum_{k_{2}=0}^{k} \sum_{k_{1}=0}^{k_{2}} B_{2}\left(k_{1}\right) S_{i}^{h}\left(k_{2}-k_{1}\right) I_{i}^{v}\left(k-k_{2}\right)\right. \\
& \quad-\mu_{h} S_{i}^{h}(k)+\lambda_{h} R_{i}^{h}(k) \\
& \left.\quad-\sum_{k_{2}=0}^{k} \sum_{k_{1}=0}^{k_{2}} B_{1}\left(k_{1}\right) S_{i}^{h}\left(k_{2}-k_{1}\right) I_{i}^{h}\left(k-k_{2}\right)\right),
\end{aligned}
$$

$I_{i}^{h}(k+1)$

$$
=\frac{\Gamma(\alpha k+1)}{\Gamma(\alpha(k+1)+1)}
$$$$
\times\left(\sum_{k_{2}=0}^{k} \sum_{k_{1}=0}^{k_{2}} B_{2}\left(k_{1}\right) S_{i}^{h}\left(k_{2}-k_{1}\right) I_{i}^{v}\left(k-k_{2}\right)\right.
$$$$
-\left(\mu_{h}+\delta_{h}+\gamma_{h}\right) I_{i}^{h}(k)
$$$$
\left.+\sum_{k_{2}=0}^{k} \sum_{k_{1}=0}^{k_{2}} B_{1}\left(k_{1}\right) S_{i}^{h}\left(k_{2}-k_{1}\right) I_{i}^{h}\left(k-k_{2}\right)\right)
$$

$R_{i}^{h}(k+1)=\frac{\Gamma(\alpha k+1)}{\Gamma(\alpha(k+1)+1)}\left(\gamma_{h} I_{i}^{h}(k)-\left(\mu_{h}+\lambda_{h}\right) R_{i}^{h}(k)\right)$, 
TABLE 1: The relation between the original function and the differential transform.

\begin{tabular}{lc}
\hline The original function & The transformed function \\
\hline$g(t)=x(t)+y(t)$ & $G(k)=X(k)+Y(k)$ \\
\hline$g(t)=\alpha x(t)$ & $G(k)=\alpha X(k)$ \\
$g(t)=x(t) y(t)$ & $G(k)=\sum_{l=0}^{k} X(l) Y(k-l)$ \\
\hline$g(t)=\frac{d x(t)}{d(t)}$ & $G(k)=(k+1) X(k+1)$ \\
\hline$g(t)=\frac{d^{m} x(t)}{d t^{m}}$ & $G(k)=(k+1)(k+2) \cdots(k+m) X(k+m)$ \\
\hline
\end{tabular}

$$
\begin{aligned}
S_{i}^{v}(k+1)= & \frac{\Gamma(\alpha k+1)}{\Gamma(\alpha(k+1)+1)} \\
\times & \left(b_{2}-\gamma_{v} S_{i}^{v}(k)\right. \\
& \left.\quad-\sum_{k_{2}=0}^{k} \sum_{k_{1}=0}^{k_{2}} B_{3}\left(k_{1}\right) S_{i}^{v}\left(k_{2}-k_{1}\right) I_{i}^{h}\left(k-k_{2}\right)\right), \\
I_{i}^{v}(k+1)= & \frac{\Gamma(k+1)}{\Gamma(\alpha(\alpha k+1)+1)} \\
& \times\left(\sum_{k_{2}=0}^{k} \sum_{k_{1}=0}^{k_{2}} B_{3}\left(k_{1}\right) S_{i}^{v}\left(k_{2}-k_{1}\right) I_{i}^{h}\left(k-k_{2}\right)\right. \\
& \left.\quad-\left(\gamma_{v}+\delta_{v}\right) I_{i}^{v}(k)\right),
\end{aligned}
$$

such that $S_{i}^{h}\left(t_{i-1}\right)=S_{i-1}^{h}\left(t_{i-1}\right), I_{i}^{h}\left(t_{i-1}\right)=I_{i-1}^{h}\left(t_{i-1}\right), R_{i}^{h}\left(t_{i-1}\right)=$ $R_{i-1}^{h}\left(t_{i-1}\right), S_{i}^{v}\left(t_{i-1}\right)=S_{i-1}^{v}\left(t_{i-1}\right)$, and $I_{i}^{v}\left(t_{i-1}\right)=I_{i-1}^{v}\left(t_{i-1}\right)$. Initially starting from $S_{0}^{h}=e_{1}, I_{0}^{h}=e_{2}, R_{0}^{h}=e_{3}, S_{0}^{v}=e_{4}$, and $I_{0}^{v}=e_{5}$, with the use of (14). The solution can be obtained by the MSGDTM given in (13). Table 1 represents the relation between the original function and the differential transform.

\section{Numerical Results}

In this section, we present the numerical solution of the proposed model (3) numerically by the classical RungeKutta order four method for the integer case derivative and analytically we use the MSGDTM. We apply the algorithm on the interval $[0,30]$ for the approximate solution of nonlinear fractional differential equations (3). For $K=10$ and $M=$ 3000 , the results are obtained. The results are obtained by using computer algebra package Mathematica. The initial conditions $e_{1}=40, e_{2}=20, e_{3}=10, e_{4}=50$, and $e_{5}=30$ are used. The values for the parameters we assumed are presented in Table 2. In Figures 1, 2, 3, 4, and 5, the approximate solution is obtained by MSGDTM and classical Runge-Kutta method order four scheme for $\alpha=1$. The approximate solution of MSGDTM has good agreement with

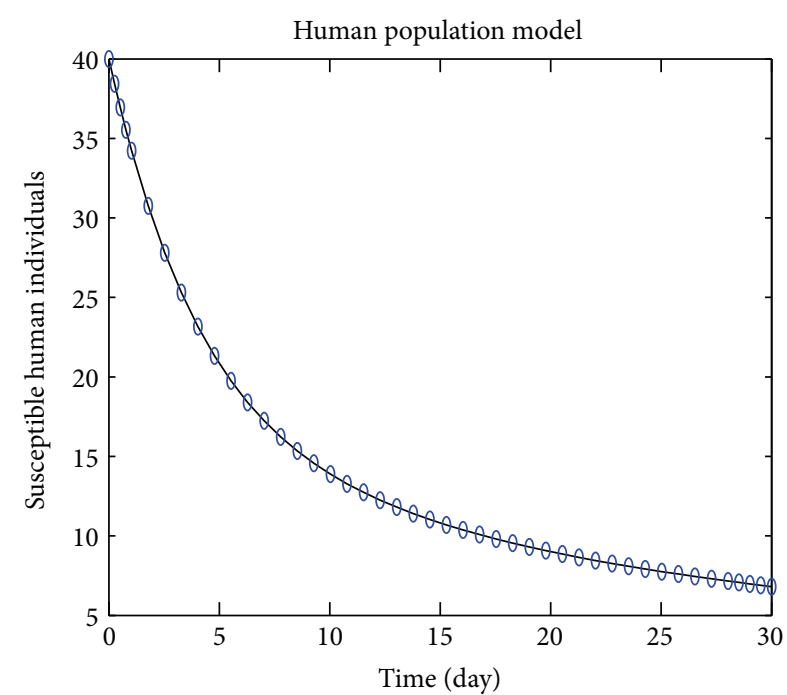

FIGURE 1: The dotted line and the solid line connectively show the Runge-Kutta method and MSGDTM.

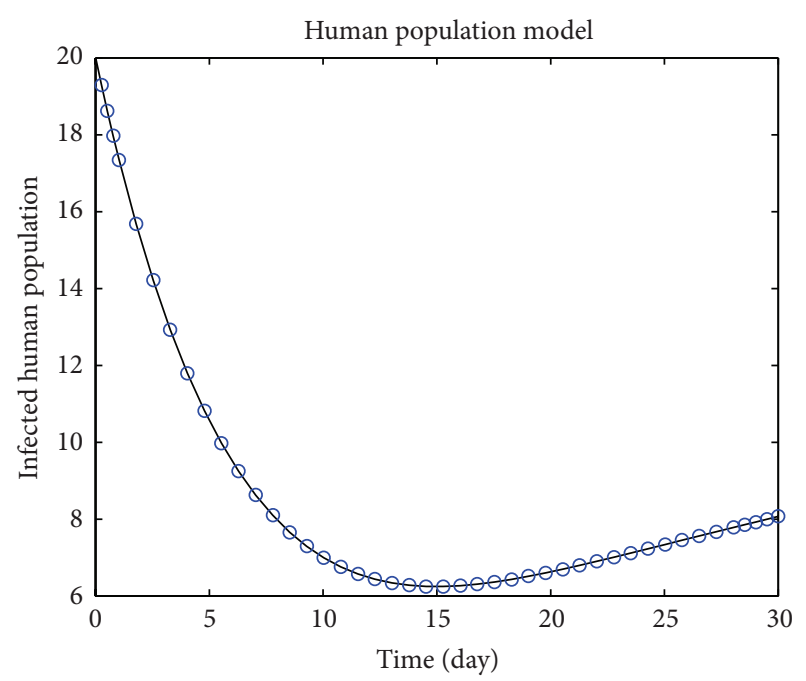

FIGURE 2: Comparison of RK method (dotted line) and MSGDTM (solid line).

the Runge-Kutta order four method. Figures 6, 7, 8, 9, and 10 represent the approximate solution obtained by MSGDTM and classical Runge-Kutta order four scheme considering different values of $\alpha$. We conclude from our graphical results that the MSGDTM results behave as closely as Runge-Kutta method.

\section{Conclusion}

In this paper, we have presented an approximate solution to an epidemic model of leptospirosis in fractional order. First, we obtained the analytical solution to the fractional order of epidemic model. Then, we solved the fractional order 
TABLE 2: Parameter values used in the numerical simulations of the optimal control.

\begin{tabular}{lccc}
\hline Notation & Parameter description & Value & Reference \\
\hline$b_{1}$ & Recruitment rate for human & 1.6 & {$[11]$} \\
$\lambda_{h}$ & Proportionality constant & 0.066 & {$[30]$} \\
$\mu_{h}$ & Natural death rate of human & $4.6 \times 10^{-5}$ & {$\left[1.8 \times 10^{-3}\right.$} \\
$\gamma_{v}$ & Natural death rate of vector & $1.0 \times 10^{-5}$ & {$[11]$} \\
$\delta_{h}$ & Death rate due to disease at human class & $2.7 \times 10^{-3}$ & {$[31]$} \\
$\gamma_{h}$ & Recovery rate of the infection & 1.9 & Assumed \\
$b_{2}$ & Birth rate of vector & 0.0089 & Assumed \\
$\beta_{2}$ & Transmission between $S^{h}$ and $I^{v}$ & 0.0079 & Assumed \\
$\beta_{3}$ & Transmission between $S^{v}$ and $I^{h}$ & 0.00013 & Assumed \\
$\beta_{1}$ & Transmission coefficient between $S^{h}$ and $I^{h}$ & 0.0027 & {$[11]$} \\
$\gamma_{v}$ & Natural death rate of vector & & \\
\hline
\end{tabular}

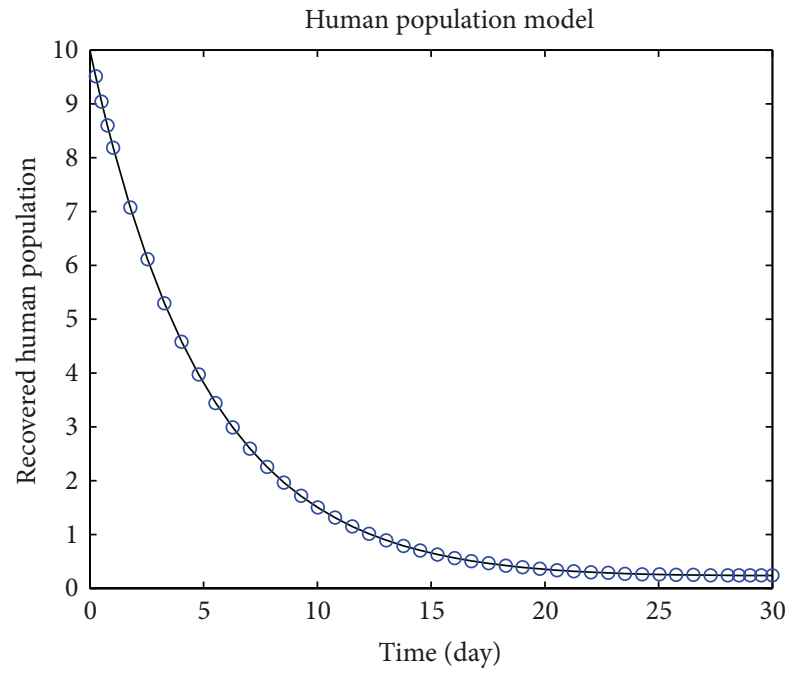

FIGURE 3: Comparison of RK method (dotted line) and MSGDTM (solid line).

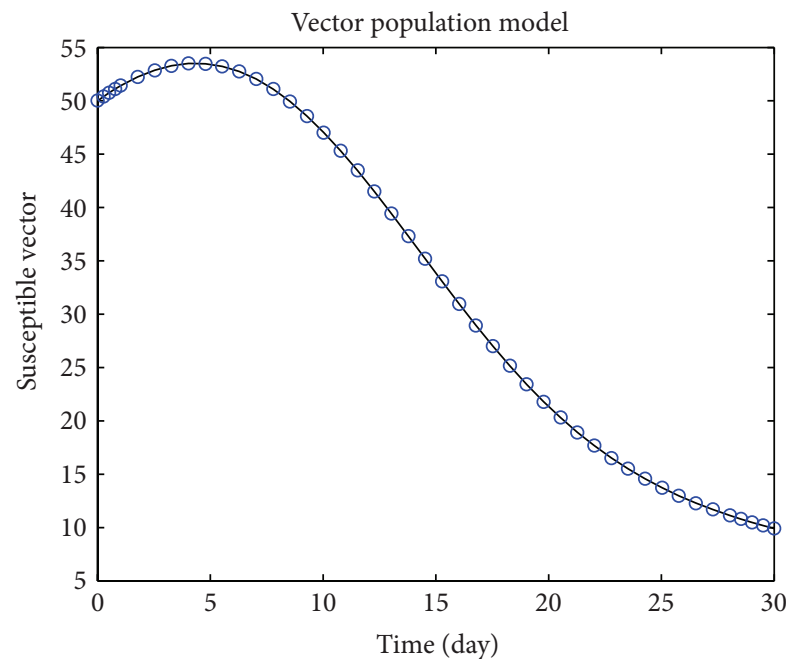

FIgURE 4: Comparison of RK method (dotted line) and MSGDTM (solid line).

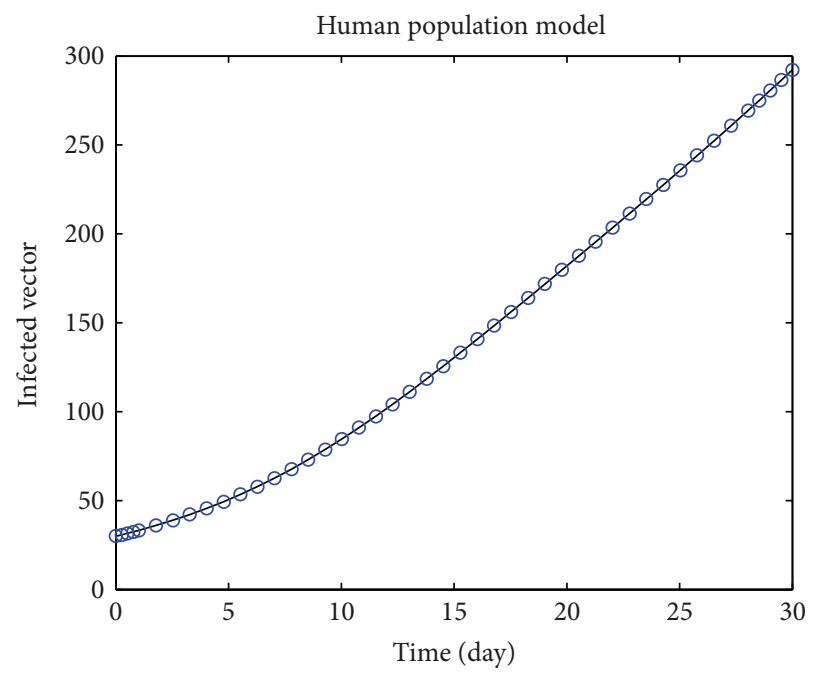

FIGURE 5: Comparison of RK method (dotted line) and MSGDTM (solid line).

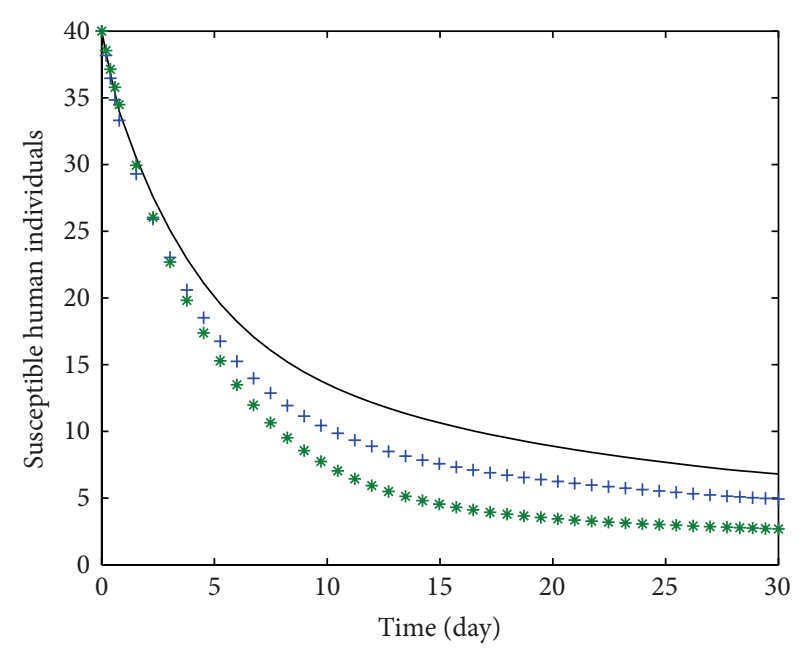

FIGURE 6: Susceptible human: solid line $\alpha=1.0,++$ line $(\alpha=0.95)$, and $* *$ line $(\alpha=0.90)$. 


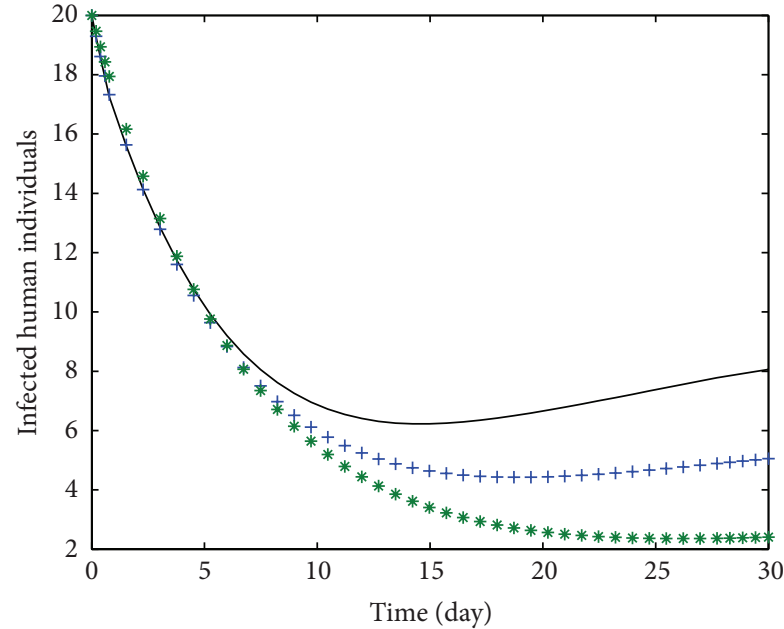

FIGURE 7: Infected human: solid line $\alpha=1.0,++$ line $(\alpha=0.95)$, and $* *$ line $(\alpha=0.90)$.

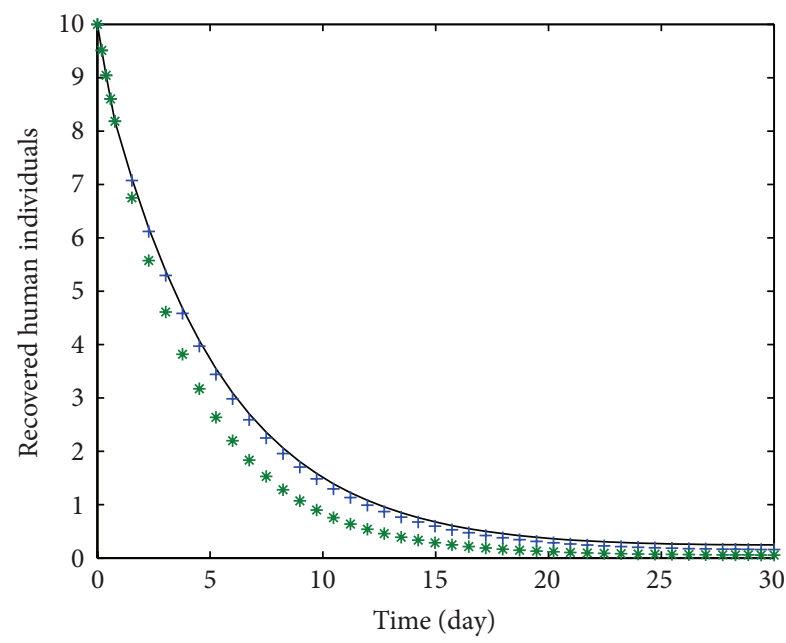

FIGURE 8: Recovered human: solid line $\alpha=1.0,++$ line $(\alpha=0.95)$, and $* *$ line $(\alpha=0.90)$

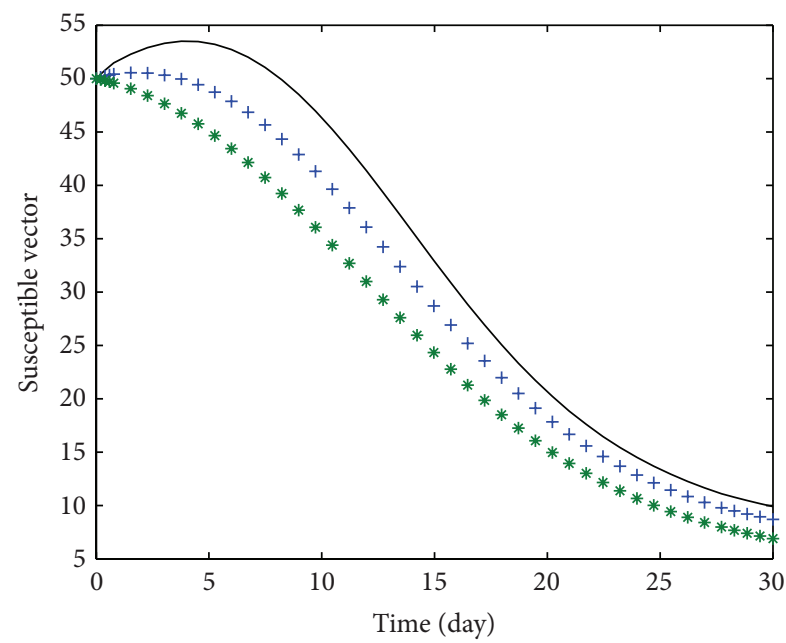

FIGURE 9: Susceptible vector: solid line $\alpha=1.0,++$ line $(\alpha=0.95)$, and $* *$ line $(\alpha=0.90)$.

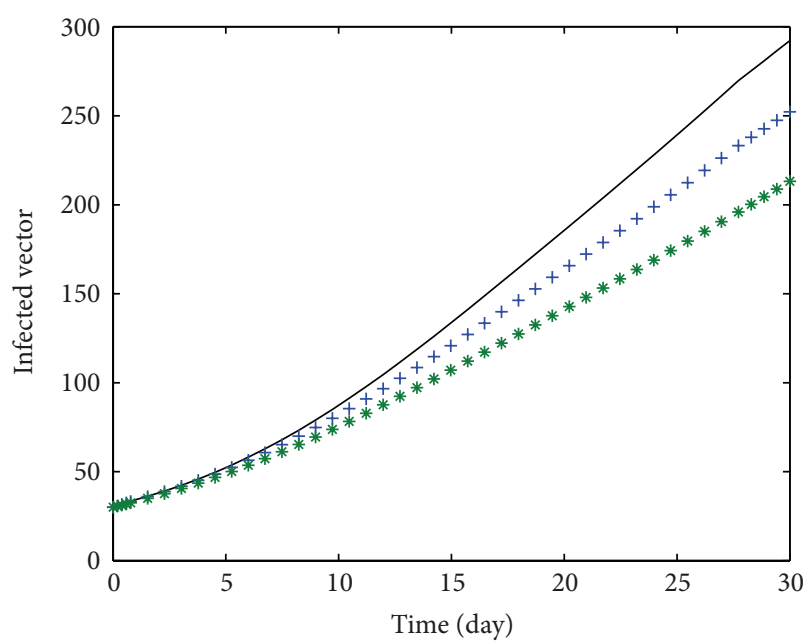

Figure 10: Infected vector: solid line $(\alpha=1.0,++$ line $(\alpha=0.95)$, and $* *$ line $(\alpha=0.90)$.

system and the results were compared with classical RungeKutta method. The results obtained from MGDTM have a good agreement with Runge-Kutta order four method. The MGDTM results are valid for a larger $t$.

\section{Conflict of Interests}

The authors declare that there is no conflict of interests regarding publication of the paper.

\section{Acknowledgments}

The authors are thankful to handling editor and the anonymous reviewers for their careful reading and suggestions that greatly improved the presentation of this paper. The authors are grateful to Universiti Teknologi PETRONAS for the financial support.

\section{References}

[1] http://www.cdc.gov/leptospirosis/.

[2] J. S. Leszczynski, An Introduction to Fractional Mechanics, Czestochowa University of Technology, Czestochowa, Poland, 2011.

[3] R. L. Magin, Fractional Calculus in Bioengineering, Begell House, Redding, Mass, USA, 2006.

[4] Y. Khan, Q. Wu, N. Faraz, A. Yildirim, and M. Madani, "A new fractional analytical approach via a modified RiemannLiouville derivative," Applied Mathematics Letters, vol. 25, no. 10, pp. 1340-1346, 2012.

[5] F. A. Abdullah, "Using fractional differential equations to model the Michaelis-Menten reaction in a 2-d region containing obstacles," ScienceAsia, vol. 37, no. 1, pp. 75-78, 2011.

[6] V. S. Ertürk, G. Zaman, and S. Momani, "A numeric-analytic method for approximating a giving up smoking model containing fractional derivatives," Computers and Mathematics with Applications, vol. 64, no. 10, pp. 3065-3074, 2012.

[7] V. S. Erturk, A. Yildirim, S. Momanic, and Y. Khan, "The differential transform method and Pade approximants for a fractional population growth model," International Journal of 
Numerical Methods for Heat \& Fluid Flow, vol. 22, no. 6-7, pp. 791-802, 2012.

[8] V. S. Erturk, Z. M. Odibat, and S. Momani, "An approximate solution of a fractional order differential equation model of human T-cell lymphotropic virus I (HTLV-I) infection of CD4 ${ }^{+}$ T-cells," Computers \& Mathematics with Applications, vol. 62, no. 3, pp. 996-1002, 2011.

[9] A. Alawneh, "Application of the multistep generalized differential transform method to solve a time-fractional enzyme kinetics," Discrete Dynamics in Nature and Society, vol. 2013, Article ID 592938, 7 pages, 2013.

[10] Y. Khan, N. Faraz, A. Yildirim, and Q. Wu, "Fractional variational iteration method for fractional initial-boundary value problems arising in the application of nonlinear science," Computers \& Mathematics with Applications, vol. 62, no. 5, pp. 22732278, 2011.

[11] G. Zaman, M. Altaf Khan, S. Islam, M. I. Chohan, and I. H. Jung, "Modeling dynamical interactions between leptospirosis infected vector and human population," Applied Mathematical Sciences, vol. 6, no. 25-28, pp. 1287-1302, 2012.

[12] M. A. Khan, G. Zaman, S. Islam, and M. I. Chouhan, "Optimal campaign in leptospirosis epidemic by multiple control variables," Applied Mathematics, vol. 3, no. 11, pp. 1655-1663, 2012.

[13] S. F. Saddiq, M. A. Khan, S. Islam, G. Zaman, N. Khalid, and S. I. A. Shah, "Optimal control of an epidemic model of leptospirosis with time delay," Life Science Journal, vol. 10, no. 3, pp. 292-298, 2013.

[14] G. Zaman, "Dynamical behavior of leptospirosis disease and role of optimal control theory," International Journal of Computer Mathematics, vol. 7, article J10, 2010.

[15] M. A. Khan, S. Islam, and S. A. Khan, "Mathematical modeling towards the dynamical interaction of leptospirosis," Applied Mathematics and Information Sciences, vol. 8, no. 3, pp. 10491056, 2014.

[16] M. A. Khan, S. Islam, S. A. Khan, and G. Zaman, "Global stability of vector-host disease with variable population size," BioMed Research International, vol. 2013, Article ID 710917, 9 pages, 2013.

[17] Y. Khan, M. Fardi, K. Sayevand, and M. Ghasemi, "Solution of nonlinear fractional differential equations using an efficient approach," Neural Computing and Applications, vol. 24, no. 1, pp. 187-192, 2014.

[18] Z. Odibat, S. Momani, and V. S. Erturk, "Generalized differential transform method: application to differential equations of fractional order," Applied Mathematics and Computation, vol. 197, no. 2, pp. 467-477, 2008.

[19] S. Momani and Z. Odibat, "A novel method for nonlinear fractional partial differential equations: combination of DTM and generalized Taylor's formula," Journal of Computational and Applied Mathematics, vol. 220, no. 1-2, pp. 85-95, 2008.

[20] Z. Odibat and S. Momani, "A generalized differential transform method for linear partial differential equations of fractional order," Applied Mathematics Letters, vol. 21, no. 2, pp. 194-199, 2008.

[21] Z. M. Odibat, C. Bertelle, M. A. Aziz-Alaoui, and G. H. Duchamp, "A multi-step differential transform method and application to non-chaotic or chaotic systems," Computers \& Mathematics with Applications, vol. 59, no. 4, pp. 1462-1472, 2010.

[22] I. Podlubny, Fractional Differential Equations, Academic Press, New York, NY, USA, 1999.
[23] A. A. Kilbas, H. M. Srivastava, and J. Trujillo, Theory and Applications of Fractional Differential Equations, Elsevier, Amsterdam, The Netherlands, 2006.

[24] S. Das, Functional Fractional Calculus, Springer, 2011.

[25] Z. M. Odibat and N. T. Shawagfeh, "Generalized Taylor's formula," Applied Mathematics and Computation, vol. 186, no. 1, pp. 286-293, 2007.

[26] W. Lin, "Global existence theory and chaos control of fractional differential equations," Journal of Mathematical Analysis and Applications, vol. 332, no. 1, pp. 709-726, 2007.

[27] V. S. Erturk, S. Momani, and Z. Odibat, "Application of generalized differential transform method to multi-order fractional differential equations," Communications in Nonlinear Science and Numerical Simulation, vol. 13, no. 8, pp. 1642-1654, 2008.

[28] S. Momani, A. Freihat, and M. AL-Smadi, "Analytical study of fractional-order multiple chaotic FitzHugh-Nagumo neurons model using multistep generalized differential transform method," Abstract and Applied Analysis, vol. 2014, Article ID 276279, 10 pages, 2014.

[29] S. Bushnaq, B. Maayah, S. Momani, and A. Alsaedi, "A reproducing kernel Hilbert space method for solving systems of fractional integrodifferential equations," Abstract and Applied Analysis, vol. 2014, Article ID 103016, 6 pages, 2014.

[30] W. Triampo, D. Baowan, I. M. Tang, N. Nuttavut, J. WongEkkabut, and G. Doungchawee, "A simple deterministic model for the spread of leptospirosis in Thailand," International Journal of Biomedical Science, vol. 2, pp. 22-26, 2007.

[31] W. Tangkanakul, H. L. Smits, S. Jatanasen, and D. A. Ashford, "An emerging health problem in Thailand," The Southeast Asian Journal of Tropical Medicine and Public Health, vol. 36, pp. 281288, 2005. 


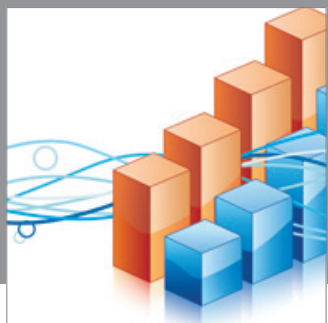

Advances in

Operations Research

mansans

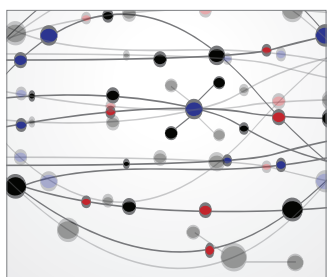

The Scientific World Journal
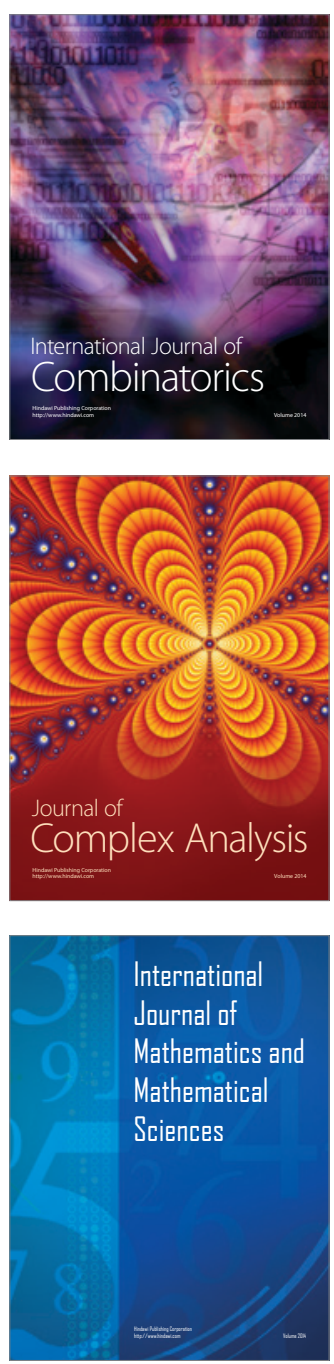
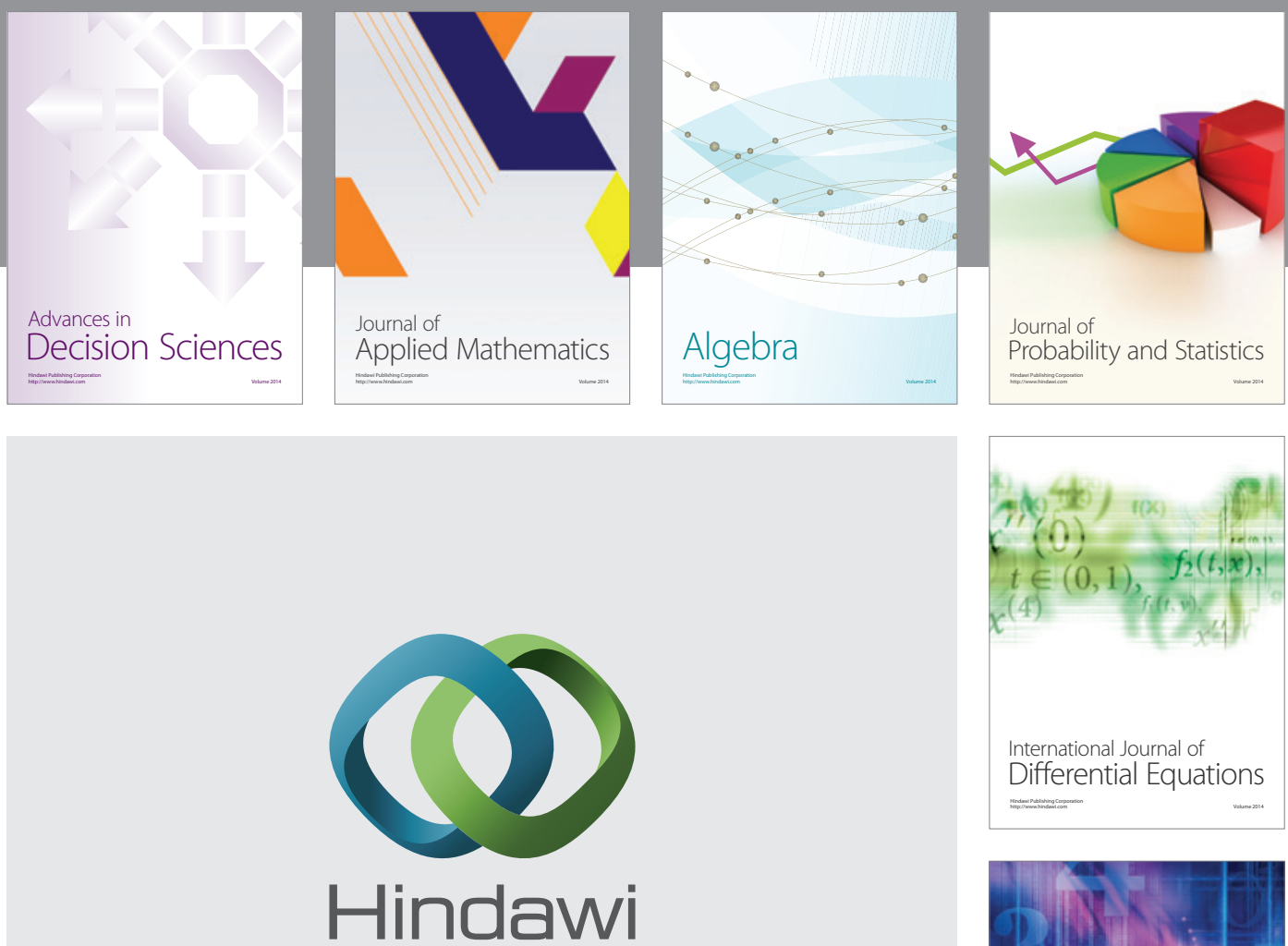

Submit your manuscripts at http://www.hindawi.com
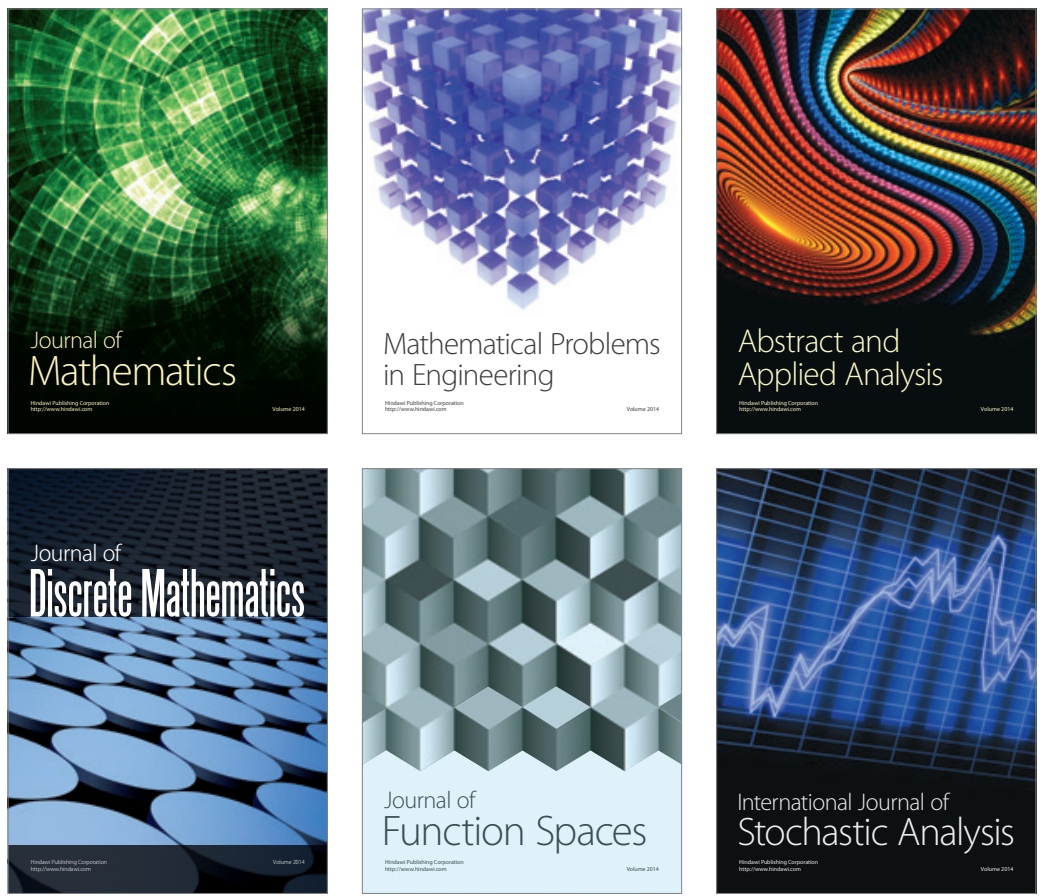

Journal of

Function Spaces

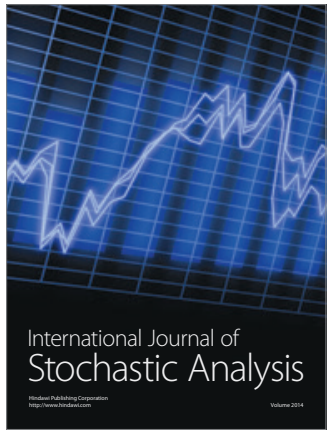

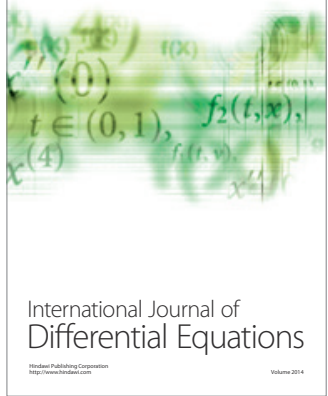
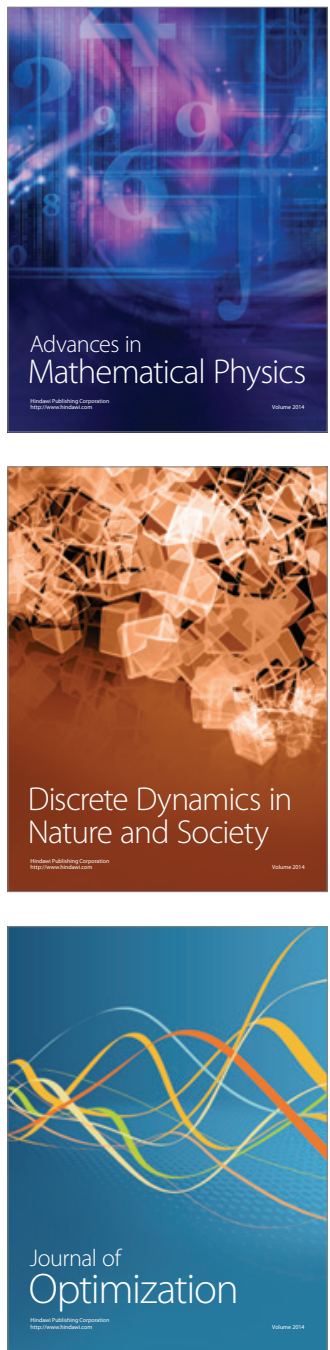